BULLETIN Bulletin hispanique

HISPANIQUE Université Michel de Montaigne Bordeaux

$114-2 \mid 2012$

Varia

Jean-Louis Guereña, Un infierno español. Un ensayo de bibliografía de publicaciones eróticas españolas clandestinas (1812-1939)

Libris, Madrid, 2011

Jean-François Botrel

(2) OpenEdition

Journals

Édition électronique

URL : https://journals.openedition.org/bulletinhispanique/2301

DOI : 10.4000/bulletinhispanique.2301

ISSN : $1775-3821$

Éditeur

Presses universitaires de Bordeaux

Édition imprimée

Date de publication : 31 décembre 2012

Pagination : $978-980$

ISBN : 978-2-86781-855-4

ISSN : 0007-4640

Référence électronique

Jean-François Botrel, « Jean-Louis Guereña, Un infierno español. Un ensayo de bibliografía de

publicaciones eróticas españolas clandestinas (1812-1939) », Bulletin hispanique [En ligne], 114-2 | 2012, mis en ligne le 23 juillet 2013, consulté le 28 juillet 2022. URL : http://journals.openedition.org/

bulletinhispanique/2301; DOI : https://doi.org/10.4000/bulletinhispanique.2301

Ce document a été généré automatiquement le 28 juillet 2022.

Tous droits réservés 


\section{Jean-Louis Guereña, Un infierno español. Un ensayo de bibliografía de publicaciones eróticas españolas clandestinas (1812-1939)}

Libris, Madrid, 2011

Jean-François Botrel

\section{RÉFÉRENCE}

Jean-Louis Guereña, Un infierno español. Un ensayo de bibliografía de publicaciones eróticas españolas clandestinas (1812-1939), Madrid, Libris, 2011, 382 pages + 54 illustrations. ISBN(10): 84-9322-459-6/ISBN(13) 978-84-9322-459-2.

1 L'Enfer n'existait pas en Espagne -au moins à la Biblioteca Nacional-: Jean-Louis Guereña l'a inventé. Au sens où, tel un archéologue du désir, par un patient travail de collection de livres, brochures et autres suites d'images (ou de leurs références) qui auraient dû y être condamnés, il a pu réunir, pour la période qui va de 1812 (date de publication de Teresa filósofa à Bordeaux) à la fin de la Guerre Civile, un corpus de plus de 200 titres qui est ici présenté, décrit et mis en perspective au regard de la littérature et des pratiques culturelles d'hier et d'aujourd'hui.

2 À l'instar d'un Robert Lehmann-Nistche au début du XX $\mathrm{X}^{\mathrm{e}}$ siècle en Argentine, avec sa collecte de bordellpoesie ${ }^{1}$, Jean-Louis Guereña a, dans le cadre d'une histoire sociale et culturelle de l'Espagne des $\mathrm{XIX}^{\mathrm{e}}$ et $\mathrm{XX}^{\mathrm{e}}$ siècles, choisi d'explorer un domaine éditorial que les libraires spécialisés qualifient euphémistiquement de curiosa et dont les contenus peuvent être qualifiés d'érotiques, de pornographiques, ou tout simplement de verdes, mais non de galantes, picantes ou frescos, comme telle «colección de cuentecillos más frescos que la lechuga », intitulée Pimientos picantes, des publications qui ne semblent pas avoir été mieux conservées dans les bibliothèques publiques. Les 
qualificatifs de l'époque ne correspondent d'ailleurs pas toujours à ceux aujourd'hui utilisés et, dans ce domaine plus que dans tout autre, les frontières qui permettent d'inclure ou d'exclure peuvent être subjectives ou arbitraires: Guereña a choisi (cf. p. 21) de se limiter aux publications clandestines (ou figurant dans des catalogues clandestins), comprenant des illustrations explicitement pornographiques, et de prendre en compte les publications scatologiques.

3 Étant donné leurs modes de production (souvent clandestine, dans des collections aussi multiples qu'éphémères), de diffusion (sous le manteau), de consommation (secrète ou confidentielle, pour des imprimés « qu'on-ne-lit-que-d'une-main »), et de conservation (" du deuxième rayon »), ces publications qui sont majoritairement des brochures de petit format et de peu de pages (une trentaine) sont, malgré des tirages relativement abondants, devenues rares et d'autant plus recherchées. Seuls quelques chercheurs comme Cerezo, Blas Vega, Infantes, Cátedra, Dutel, López Barbadillo ou Rose, ont choisi de leur donner une publicité dont elles se gardaient bien à l'origine, en les répertoriant et, plus rarement, en les donnant à voir et à lire.

C'est ce à quoi s'emploie Jean-Louis Guereña dont les premières publications dans ce domaine remontent à 1999, et qui, à la différence d'un Henry Spencer Ashbee (1834-1900) ou de Cela, par exemple, leur applique un regard totalement distancié et quasiment clinique. On peut l'apprécier dans les descriptions bibliographiques qui répondent aux normes les plus exigeantes en la matière : on trouvera, bien sûr, toutes les indications sur les auteurs ou leurs alias souvent cocasses ou salaces (Doctor Coñocida, Telometo Porelano, Casto Cascósela y Pingalisa, Cupidín, Polla dura/tiesa/de oro, etc.), sur les titres plus ou moins suggestifs ou explicites (de A la caza del coño a $E l$ yate de los placeres), sur les lieux d'édition réels ou supposés et parfois délibérément imaginaires (Itapicuá, Fornicópolis, Sífilis, etc.), les éditeurs ou imprimeurs (comme Antonio Astiazarain, officiellement établi à Tarbes et le plus prolifique dans les années 1920, mais aussi l'Imprenta de Priapo, a cargo de Mercurio, P. K. Rajo de L. Fante, etc.), sur la pagination, les formats, et l'appartenance éventuelle à une collection (Biblioteca Virgo, Colección Afrodita, Los Maestros de la lujuria, Colecció Col.lonuda ou encore la mystérieuse Biblioteca $\mathrm{H}$, etc.). A ces indications rarement réunies lorsqu'il s'agit de non-livres ou de publications dites mineures, Guereña a pris le soin d'ajouter, pour chacune des publications, outre un résumé sans fard du contenu, des notes très érudites qui constituent autant de fiches de traçabilité permettant de reconstituer l'histoire de l'œuvre et, parfois, de l'exemplaire, mais aussi de discuter ou de rectifier la doxa en vigueur. De même, Guereña prend soin de donner le détail des illustrations constitutives du genre, particulièrement abondantes et explicites (certains ouvrages ne sont d'ailleurs constitués que de planches ou de cartes à jouer qui - cela étonne - ne semblent pas avoir eu recours à la photographie), et d'en reproduire une cinquantaine, dont 35 en couleurs - "de subido color », comme on dit en espagnol. Le moins qu'on puisse dire, c'est que toutes ne sont pas, comme celle de la page 308 (signée Joder), dignes de Cimboldo et que tous les illustrateurs n'ont pas la patte d'un Eusebi Planas, qui est l'auteur de cartes postales pornographiques comme El noble arte del billar ( $\left.\mathrm{n}^{\circ} 140\right)$ ou Noble juego del tresillo $\left(\mathrm{n}^{\circ} 141\right)$, mais aussi des illustrations de la version pornoparodique de la pièce de Camprodón, Flor de un día ( $\left.\mathrm{n}^{\circ} 93\right)$, également illustrée par lui dans sa version romancée.

Dans cette littérature en prose narrative plus qu'en vers ou sous forme dramatique et, pour une grande partie, éminemment graphique, donc, on trouve, comme cela est 
fréquent en Espagne, beaucoup de reproductions, traductions ou adaptations d'œuvres d'auteurs français ou supposés tels - c'est apparemment un attrait et une garantie -, qu'il s'agisse de Restif de la Bretonne ( $\left.n^{\circ} 18\right)$, de Voltaire $\left(n^{\circ} 74\right)$, de Diderot $\left(n^{\circ} 183\right)$, de Pigault-Lebrun ( $\left.n^{\circ} 105\right)$, de la Marquise de Mannoury d'Ectot ( $\left.{ }^{\circ} 146\right)$, ou de Louise Dormienne $\left(\mathrm{n}^{\circ} 38\right)$, par exemple. On y trouve des classiques apparemment disponibles un peu partout, comme Thérèse philosophe, Le rideau levé (La cortina corrida), traditionnellement attribué à Mirabeau, ou Le portier des Chartreux (El portero de los cartujos) de Jean-Gervaise de Latouche ( $\left.\mathrm{n}^{\circ} 168\right)$, dont Guereña répertorie huit éditions. La production espagnole au sens strict (y compris en catalan) n'est évidemment pas absente: de la plus ancienne, avec la réédition du Cancionero de obras de burlas provocantes a risa de 1519, conservé à la British Library, à l'Arte de las putas de Moratín en passant par les Gracias y desgracias del ojo del culo, attribuées, comme on sait, à Quevedo, ou Samaniego $\left(n^{\circ} 10\right)$, à qui, apparemment, on ne demanda pas son avis. Un certain nombre d'auteurs contemporains devenus classiques sont également l'objet (ou les victimes) de porno-parodies, comme Espronceda dont le Diablo mundo inspire une " parodia cachonda » à un certain Alejo de Montado « Miembro robusto y erguido de la "Sociedad Virguera" del Olimpo, etc. ») ou Campoamor (avec un Nuevo tren expreso, $\mathrm{n}^{\circ}$ 151), de même que des œuvres à succès de l'époque, comme La dama de las camelias ( $\left.n^{\circ} 65\right)$, La Gran Vía ( $\left.n^{\circ} 100\right)$, La verbena de la Paloma $\left(n^{\circ} 224\right)$ ou le Don Juan Tenorio de Zorrilla $\left(\mathrm{n}^{\circ} 72, \mathrm{n}^{\circ} 200\right)$, déjà abordé sous cet aspect - on s'en souvient - par Carlos Serrano dans son Carnaval en noviembre (1996). Il faut ajouter à cela, un fort courant anticlérical, en grande partie traditionnel, présent, par exemple, dans La Torre de San Esteban o el Seminario de Venus ( $\left.\mathrm{n}^{\circ} 202\right)$, mais qui s'applique ici plus particulièrement au Padre Claret, auquel on prête la paternité des Aventuras de un pollo (n $\left.{ }^{\circ} 24\right)$ ou dont on utilise La llave de oro à l'usage des confesseurs pour une nouvelle version, avec interpolation de «dibujos y figuras las más obscenas y con instrucciones y explicaciones las más repugnantes y escandalosas» comme l'écrit le P.P. Cristóbal Fernández, C. M. F. cité par Guereña, page 247 ; une dimension présente jusque dans les appellations des imprimeries (Establecimiento Jodeográfico Ultra-Montano, par exemple) et qui trouve une expression comme emblématique dans l'opuscule $\mathrm{El}$ can-can o El virgo de Sor Teresa. Paso histórico, mímico, higiénico, gimnástico, bailable, jodible y epidémico en cuatro polvos en un solo acto de ocho cojones, escrito por un español más jodido que Isabel de Borbón que es cuanto se puede decir, Vaina, Imprenta de recojones, 1870, $32 \mathrm{p}$. con 4 litografías libres con su respectivo pie, 16.5 x $11 \mathrm{cms}\left(\mathrm{n}^{\circ} 33\right)$, résumé de la façon suivante: "Teresa, joven de 18 años, decide entrar en un convento para 'gozar todos los placeres del amor (...)', 'joder desde la mañana a la noche, como se acostumbra en los conventos'» (p. 88).

On pourrait dire que rien vraiment ne manque à ce livre, sauf sans doute la poésie, que le lecteur ou la lectrice intéressée et qui n'aura pu partager avec Jean-Louis Guereña le plaisir de la quête ou de l'enquête, préférera peut-être encore aller chercher dans la classique Floresta de poesías eróticas del Siglo de Oro d'Alzieu, Lissorgues et Jammes.

7 L'historien de la vie privée et de la sexualité, quant à lui, ne manquera pas d'être concerné par tout ce que peut révéler dans une perspective d'anthropologie historique, un travail qui aurait pu n'être qu'un simple catalogue de références bibliographiques: tous ces jeux verbaux annonciateurs dès la couverture de plaisirs encore cachés à l'intérieur et confirmés ou non dans le secret d'un cabinet ou d'une alcôve, toutes ces histoires ressassées et transgressives de la morale ambiante, toutes ces piètres représentations de l'intime ou de l'interdit ont, en effet, certainement alimenté un Eros 
national espagnol placé sous le signe de la censure et fait la délectation d'un nombre important d'hommes - et peut-être de femmes. Des hommes qui pouvaient aussi aller chercher dans les lupanars (Guereña a publié un très savant ouvrage à ce sujet) ou dans les salles de théâtre ${ }^{2}$, de pauvres remèdes à ce qui a pu être qualifié de "misère sexuelle». Sauf quelques mentions conservées sur tel ou tel exemplaire («Encarnado y verde » pour qualifier El portero de los cartujos, par exemple, p. 279), ou le fait que, sous le Franquisme, on ait encore pu faire une copie dactylographiée de Con la polla en la mano ( $\left.\mathrm{n}^{\circ} 52\right)$, on ne dispose néanmoins que de peu d'indications sur les motivations et les modes d'appropriation des lecteurs. On peut évidemment les imaginer...

Il faut donc remercier Jean-Louis Guereña d'avoir mis à jour la partie la plus " extrême » de publications appartenant au patrimoine bibliographique de l'Espagne et qui, faute d'un Enfer institué (souvent dénoncé là où il existait, mais dont les flammes ne sont finalement que métaphoriques!), aurait pu rester inaperçu, et il faut également être reconnaissant à l'Asociación de Libreros de Viejo d'avoir publié ce beau travail dans une de ses éditions non vénales (tirée à 1000 exemplaires), la $21^{\mathrm{e}} \mathrm{d}^{\mathrm{u}}$ une série initiée en 1990 : cet ouvrage, en principe destiné aux collectionneurs ou amateurs de curiosa, présente - on a pu s'en rendre compte - un intérêt qui va bien au delà de la marginalité supposée du sujet, pour les historiens du livre et de la culture, mais aussi gageons-le - pour beaucoup d'autres lecteurs curieux...

\section{NOTES}

1. Cf. Miguel A. García, Gloria B. Chicote, Voces de Tinta. Estudio preliminar y antología comentada de «Folklore argentino (1905)» de Robert Lehmann-Nitsche, La Plata, Edulp, 2008, p. 50.

2. Comme l'a récemment mis en lumière Serge Salaün dans une étude intitulée « Les mots et la "chose". Le théâtre "pornographique" en Espagne ", in : S. Salaün, Les spectacles en Espagne, Paris, Presses Sorbonne Nouvelle, 2011, pp. 249-266,

\section{AUTEURS}

\section{JEAN-FRANÇOIS BOTREL}

Université de Haute Bretagne/Rennes II 